\title{
Efficient calculation of coupling matrices for a decoupled FE/BIE formulation
}

\author{
Freek Boeykens* Hendrik Rogier* Jan Van Hese ${ }^{\dagger} \quad$ Jeannick Sercu ${ }^{\dagger}$ \\ Tim Boonen ${ }^{\dagger}$
}

\begin{abstract}
This paper presents an efficient method for calculating coupling matrices that occur in a decoupled hybrid finite element / boundary integral equation (FE/BIE) system. These coupling matrices represent projections from basis functions of one domain to basis functions of an other domain. Based on a technique for evaluating singular integrals over polyhedral domains, it becomes possible to calculate the projection integrals analytically, instead of relying on quadrature integration rules. It is shown that this reduces the CPU-time, whereas the accuracy remains correct up to machine precision.
\end{abstract}

\section{INTRODUCTION}

The finite element (FE) method offers the ability to model complex non-homogeneous materials and anisotropic structures, but suffers from domain truncation and approximated absorbing boundary conditions. Its effectiveness can be improved by combination with the boundary integral equation (BIE) method. This approach allows to extend the simulation domain to the full space, to enforce the Silver-Müller radiation conditions in the kernel functions of the integral equations and to couple multiple distant subdomains.

The FE/BIE method that is investigated is based on the symmetric approach from [1]. In this formulation, the FE and BIE simulation domains are decoupled, i.e. their solutions are discretised on different meshes or they may have the same mesh, but different type/order of basis functions. The coupling between neighbouring domains is enforced in the weak sense through a Robin-to-Robin map. Consequently, projections from basis functions of one domain to basis functions of an other domain have to be calculated, leading to projection submatrices in the FE/BIE system matrix.

In this paper, an efficient approach is proposed for calculating these projection matrices. In contrast to traditional Gauss quadrature rules, this technique calculates the projection integrals analytically, improving both the computation time and the accuracy.

\footnotetext{
*Department of Information Technology (INTEC), Ghent University, St. Pietersnieuwstraat 41, 9000 Ghent, Belgium. E-mail: freek.boeykens@intec.ugent.be

$\dagger$ Agilent Technologies Belgium N.V., Kortrijksesteenweg 1093 B, 9051 Sint-Denijs-Westrem, Belgium.
}

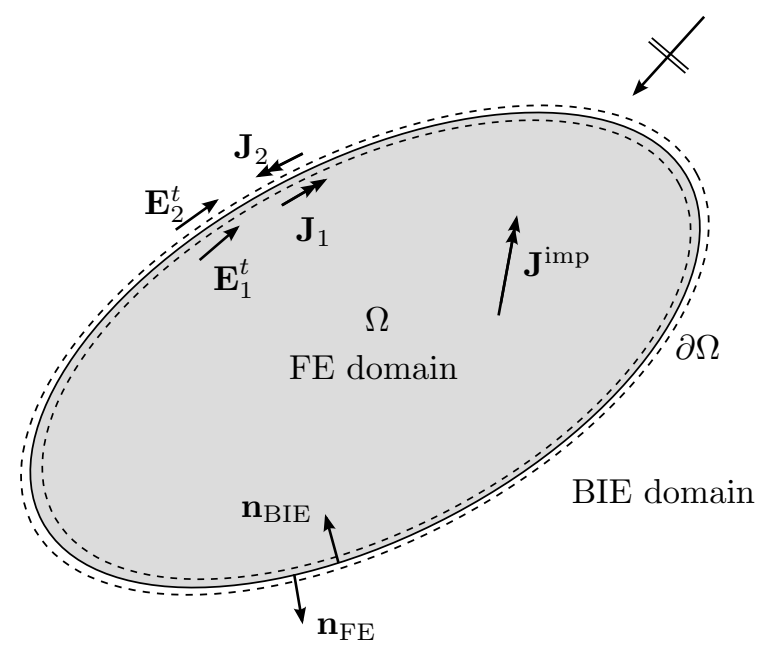

Figure 1: Problem configuration.

The organisation of this paper is as follows. The hybrid system that is investigated is described in Section 2. In Section 3 the projection calculations are explained and the conclusions are summarised in Section 4.

\section{HYBRID FE/BIE FORMULATION}

The hybrid FE/BIE formulation is based on the symmetric approach from [1], so only the matrix structure is repeated here. Since we are only interested in the coupling part, the system is shown after calculation of the Schur complement for the FE domain, eliminating all FE internal unknowns. This Schur complement is represented by $\mathcal{Y}_{1}$.

The system then becomes:

$$
\begin{array}{cccc}
\left(\begin{array}{cccc}
\mathcal{Y}_{1}+\frac{1}{2} \mathcal{P}_{3} & -\frac{1}{2} \mathcal{P}_{0} & -\frac{1}{2} \mathcal{P}_{7} & \frac{1}{2} \mathcal{P}_{1} \\
-\frac{1}{2} \mathcal{P}_{0}^{T} & -\frac{1}{2} \mathcal{P}_{4} & \frac{1}{2} \mathcal{P}_{2} & -\frac{1}{2} \mathcal{P}_{8} \\
-\frac{1}{2} \mathcal{P}_{7}^{T} & \frac{1}{2} \mathcal{P}_{2}^{T} & \frac{1}{\eta} \mathcal{T}+\frac{1}{2} \mathcal{P}_{5} & \mathcal{K} \\
\frac{1}{2} \mathcal{P}_{1}^{T} & -\frac{1}{2} \mathcal{P}_{8}^{T} & \mathcal{K}^{T} & -\eta \mathcal{T}-\frac{1}{2} \mathcal{P}_{6}
\end{array}\right) \\
& & \left(\begin{array}{c}
\mathbf{E}_{1}^{t} \\
\mathbf{J}_{1} \\
\mathbf{E}_{2}^{t} \\
\mathbf{J}_{2}
\end{array}\right)=\left(\begin{array}{c}
\mathbf{b} \\
0 \\
\mathbf{y}_{M} \\
\mathbf{y}_{E}
\end{array}\right) .
\end{array}
$$


On the BIE side, one finds the EFIE and MFIE operators $\mathcal{T}$ and $\mathcal{K}$, where the EFIE operator $\mathcal{T}$ is scaled by $\eta$, the impedance of the medium. The unknowns to be solved for are the tangential electric field and electric current at FE side: $\mathbf{E}_{1}^{t}$ and $\mathbf{J}_{1}^{t}$, and the tangential electric field and electric current at the BIE side: $\mathbf{E}_{2}^{t}$ and $\mathbf{J}_{2}$.

Galerking weighting is applied, using as expansion and test functions the curl-conforming first order edge elements [2] $\mathbf{w}_{i}$, for $\mathbf{E}_{i}^{t}$, and the divconforming RWGs [3] $\mathbf{v}_{i}$, for $\mathbf{J}_{i}$. Remark that these are $2 \mathrm{D}$ basis functions: $\mathbf{w}_{i} \in \mathrm{H}^{-1 / 2}(\operatorname{curl} ; \partial \Omega)$ and $\mathbf{v}_{i} \in \mathrm{H}^{-1 / 2}(\operatorname{div} ; \partial \Omega)$. The unknowns corresponding to the $3 \mathrm{D}$ tetrahedral $\mathrm{FE}$ basis functions have already been eliminated by the Schur complement.

The Robin-to-Robin mapping is performed by 9 projection matrices that can be subdivided into 3 groups:

1. The curl-div projections $\mathcal{P}_{0}, \mathcal{P}_{1}$ and $\mathcal{P}_{2}$ map $\mathbf{w}_{i}$ to $\mathbf{v}_{i}$.

2. The self projections $\mathcal{P}_{3}, \mathcal{P}_{4}, \mathcal{P}_{5}$ and $\mathcal{P}_{6}$ project each $\mathbf{w}_{i}$ or $\mathbf{v}_{i}$ on itself.

3. The curl-curl and div-div projections $\mathcal{P}_{7}$ and $\mathcal{P}_{8}$ map $\mathbf{w}_{1}$ on $\mathbf{w}_{2}$ and $\mathbf{v}_{1}$ on $\mathbf{v}_{2}$.

\section{PROJECTIONS}

The calculation of these projection matrices forms a crucial part in the implementation. These matrices have to be computed as efficiently as possible. Therefore, they are calculated analytically instead of using traditional Gauss quadrature rules. The calculation of these matrices is explained in this Section.

\subsection{Theory}

The projection of two vector basis functions $\mathbf{A}(\mathbf{r})$ and $\mathbf{B}(\mathbf{r})$ is given by the scalar product of the basis functions, defined as the surface integral:

$$
P=\int_{\Sigma} \mathbf{A}(\mathbf{r}) \cdot \mathbf{B}(\mathbf{r}) \mathrm{d} S=\int_{\Sigma} \Phi(\mathbf{r}) \mathrm{d} S .
$$

The integration domain $\Sigma$ is the overlap area of the support of the two basis functions and, since a triangular mesh is used, can be seen as the overlap area of two triangles. This makes $\Sigma$ a polygon spanned by maximum 6 vertices. The use of traditional Gauss quadrature rules for calculating these integrals then becomes more complicated since different polygons need different Gauss rules. Alternatively, one can also subdivide the polygon into triangles, but this slows down the calculation.

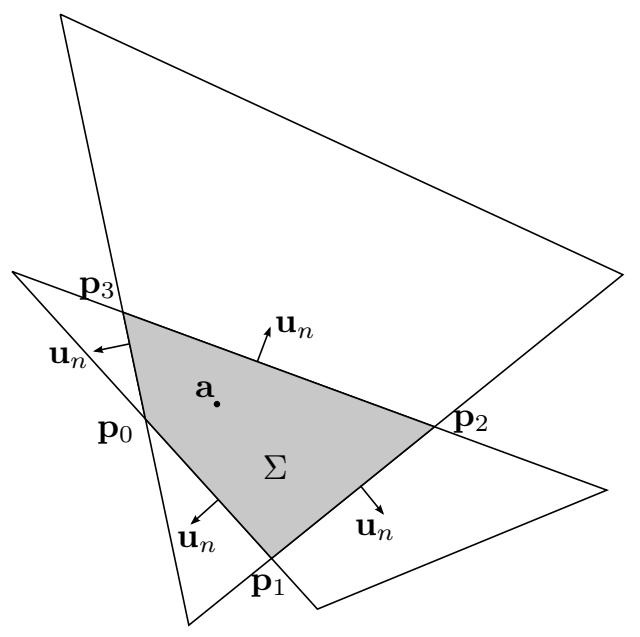

Figure 2: Triangle intersection.

It is however possible to calculate these integrals analytically, based on a technique for evaluating singular integrals over polyhedral domains. This technique, as described in [4], allows to reduce the integral of $\Phi(\mathbf{r})$ over $\Sigma$ to an integral over the boundary $\partial \Sigma$, allowing the kernel of $\Phi(\mathbf{r})$ to become singular in a point $\mathbf{a} \in \Sigma$. This is demonstrated in Figure 2. The overlap area of the two triangles is $\Sigma$ and a potentially singular point is $\mathbf{a}$.

From [4] it follows then that, if $\Phi(\mathbf{r})$ is continuously differentiable over an open domain containing the conical domain

$$
\Sigma(\mathbf{a})=\{t \mathbf{r}+(1-t) \mathbf{a}: 0<t \leq 1, \mathbf{r} \in \Sigma\},
$$

and provided that

$$
\lim _{t \rightarrow 0} t^{2} \Phi(t \mathbf{r}+(1-t) \mathbf{a})=0
$$

the following result is valid:

$$
P=\int_{\partial \Sigma}(\mathbf{r}-\mathbf{a}) \cdot \mathbf{u}_{n} \mathrm{~d} l \int_{0}^{1} t \Phi(t \mathbf{r}+(1-t) \mathbf{a}) \mathrm{d} t .
$$

With this generalised Gauss theorem, the surface integral (2) is transformed into a line integral along the edges of the overlap area. Moreover, since the scalar product of two basis functions has no singularities, conditions (3) and (4) are always satisfied.

In the example of Figure 2, this means that the projection is calculated along the lines $\mathbf{p}_{i} \mathbf{p}_{(i+1) \% 4}$ with outward normal $\mathbf{u}_{n}$ using (5). The point $\mathbf{a}$ can be chosen arbitrarily in $\Sigma$.

\subsection{Practical Implementation}

The calculation of $P$ is performed in different stages. First, the integrand $\Phi(\mathbf{r})$ is calculated. Since it is computed per triangle-triangle overlap, 
this is a $2 \mathrm{D}$ problem and hence $\Phi(\mathbf{r})$ can be written as a polynome in $x$ and $y$. A great benefit is that this is the only part in the algorithm where the scalar product of the basis functions is needed. This makes the implementation easy to extend to higher order basis functions.

Next, the inner integral of (5) is calculated. As already mentioned before, the point $\mathbf{a}$ is an arbitrary point in $\Sigma$, since $\Phi(\mathbf{r})$ has no singularities.

$$
f(\mathbf{r})=\int_{0}^{1} t \Phi(t \mathbf{r}+(1-t) \mathbf{a}) \mathrm{d} t .
$$

The projection integral then becomes:

$$
\begin{aligned}
P & =\int_{\partial \Sigma}(\mathbf{r}-\mathbf{a}) \cdot \mathbf{u}_{n} f(\mathbf{r}) \mathrm{d} S_{n} \\
& =\sum_{i}\left\|\mathbf{p}_{i+1}-\mathbf{p}_{i}\right\| I\left(\mathbf{p}_{i}, \mathbf{p}_{i+1}\right) .
\end{aligned}
$$

with

$$
I(\mathbf{p}, \mathbf{q})=(\mathbf{p}-\mathbf{a}) \cdot \mathbf{u}_{n} \int_{0}^{1} f((1-t) \mathbf{p}+t \mathbf{q}) \mathrm{d} t
$$

Remark that the index $i$ assumes cyclical values. This means that $\mathbf{p}_{n}=\mathbf{p}_{0}$, where $n$ is the number of vertices of $\Sigma$.

The integral (9) has to be calculated over each line pq with outward normal $\mathbf{u}_{n}$. Remark that by choosing a equal to a vertex of $\Sigma$, two line integrals become 0 . The calculation of (6) and (9) is performed analytically, hence consuming only a small fraction of CPU-time.

\subsection{Example}

In order to compare the analytical method with a numerical method using Gauss quadrature rules, all 9 coupling matrices $\mathcal{P}_{0}, \mathcal{P}_{1}, \ldots, \mathcal{P}_{8}$ are computed using both techniques. The numerical method with Gauss quadrature rules uses 3 evaluation points per triangle. This means that functions up to second order are correctly computed up to machine precision. All calculations are performed using an Intel Core 2 processor with 8 GB RAM, and the analytical and numerical computations are equal up to machine precision.

Figure 3 shows the CPU time that is needed to compute the matrices as a function of the number of FE unknowns when the FE and BIE domains share the same mesh. In this configuration, all overlap areas are triangles of the mesh and hence only a slight improvement in performance is observed for the analytical method over the numerical approach.

When the FE and BIE meshes are not identical, the overlap areas become polygons with maximum

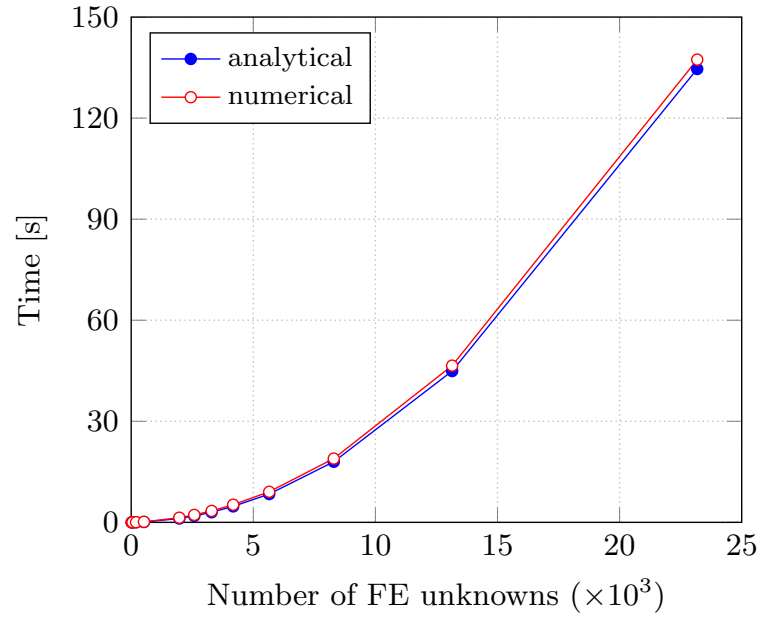

Figure 3: Computation time for the coupling matrices when the FE and BIE domains share the same mesh.

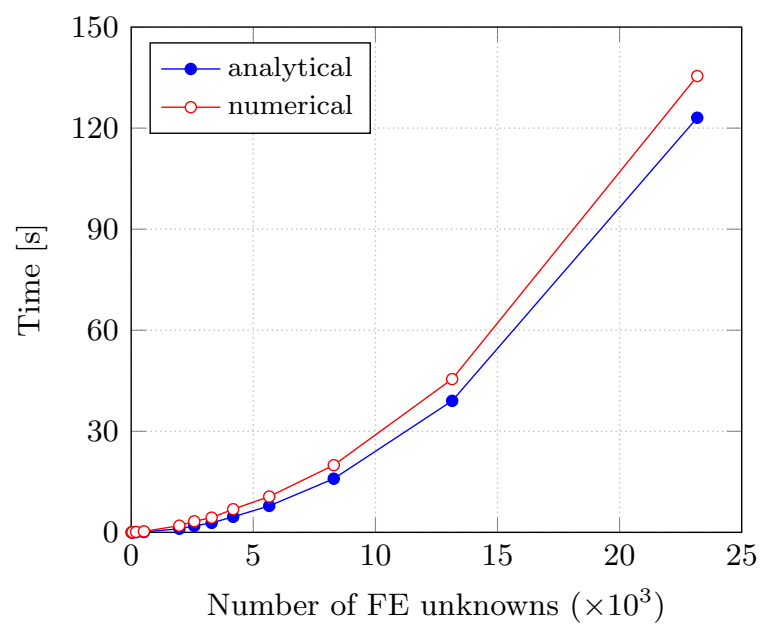

Figure 4: Computation time for the coupling matrices when the FE and BIE solutions are discretised on different meshes.

6 vertices and the analytical method becomes significantly faster than the numerical method. This is displayed in Figure 4. The numerical method calculates the projections by splitting the polygon in triangles and then applying triangular Gauss quadrature rules. In this configuration, the number of BIE unknowns is slightly smaller than the number of FE unknowns.

When higher order basis functions are used, more evaluation points per triangle are needed for the numerical approach in order to achieve the same accuracy, whereas the performance of the analyt- 


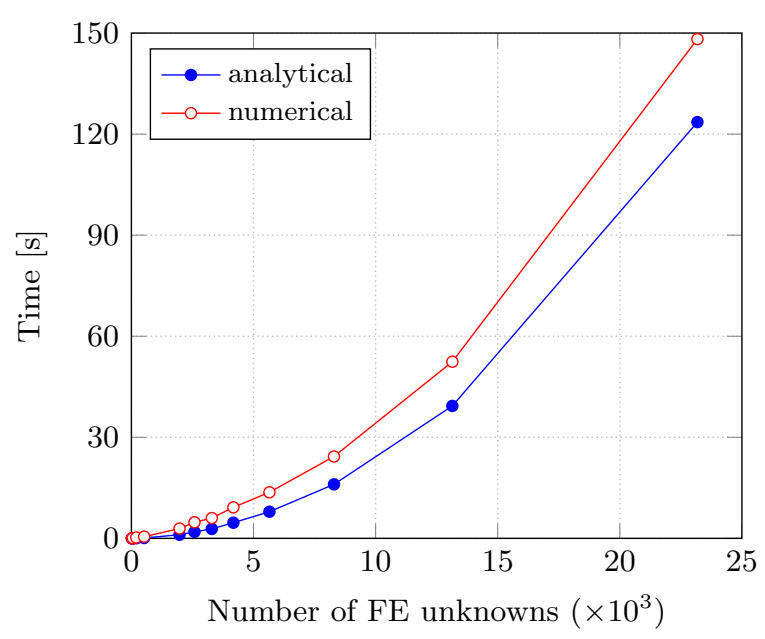

Figure 5: Computation time for the coupling matrices when the FE and BIE solutions are discretised on different meshes and 6 evaluation points per triangle are used in the numerical method.

ical method remains almost constant in terms of accuracy and simulation speed. As a last example, Figure 5 demonstrates the computation time when 6 Gauss evaluation points per triangle are used in order to calculate functions up to fourth order correctly. This is necessary when second order FE basis functions are used. The gap in computation time between the two techniques increases even more.

\section{CONCLUSION}

In this paper, an efficient method to calculate coupling matrices that occur in a hybrid FE/BIE system was presented. This method is based on a generalised Gauss theorem and allows to compute the projections analytically. A better performance compared to Gauss quadrature techniques is observed, whereas the accuracy is still correct up to machine precision.

\section{Acknowledgments}

The work of F. Boeykens was supported by a doctoral grant from the Agency for Innovation by Science and Technology in Flanders (IWT).

\section{References}

[1] K. Zhao, M. N. Vouvakis, and J.-F. Lee, "Solving electromagnetic problems using a novel symmetric fem-bem approach," IEEE Trans. Magn., vol. 42, no. 4, pp. 583-586, Apr. 2006.
[2] J.-C. Nédélec, "Mixed finite elements in R3," Numerische Mathematik, vol. 35, pp. 315-341, 1980.

[3] S. M. Rao, D. R. Wilton, and A. W. Glisson, "Electromagnetic scattering by surfaces of arbitrary shapes," IEEE Trans. Antennas Propag., vol. 30, no. 3, pp. 409-418, May 1982.

[4] L. Knockaert, "A general Gauss theorem for evaluating singular integrals over polyhedral domains," Electromagnetics, vol. 11, pp. 269-280, 1991. 